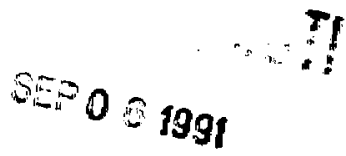

\title{
INCLUSIVE LARGE MASS MUON PAIR PRODUCTION IN ULTRA-RELATIVISTIC NUCLEUS-NUCLEUS COLLISIONS FOR COLLIDING BEAMS*
}

\author{
L. E. ROBERTS \\ Department of Physics \\ Lincoln University \\ Lincoln University, PA 19352 \\ High Energy Physics Division \\ Argonne National Laboratory \\ Argonne, IL 60439 \\ The Institute for Scientific Studies \\ Wheaton, IL 60187
}

\begin{abstract}
Abatract
For colliding beams of several species of ions we compare thermal to perturbative quantum chromodynamic contributions for inclusive large mass muon pair production by using a hydrodynamical model to estimate the temperatures of the quark-gluon plasma produced by each species. The production of high energy dimuons with $M \simeq 2-$ $4 \mathrm{GeV}$, will be favored energetically by the quark-gluon plasma.
\end{abstract}

-Work supported by the U.S. Department of Eaergy, Diviaion of High Eaergy Physics, Contract W-31-102. ENG-38. 


\section{INTRODUCTION}

In this letter we perform an updated cstimate for inclusive large mass muon pair production in ultra-relativistic nucleus-nucleus collisions. We do this for colliding beams. The motivation for doing this is that anomalously high lepton pair production will be one of the signals of the presence of the quark-gluon plasma (QGP).1,2 Another reason for doing this is that when machines such as Brookhaven's R.H.I.C. come on line, the experiments performed with them will provide a rich testing ground for our ideas about the phase transition that may occur in ultra-relativistic nucleus-nucleus collisions.

Several methods exist for treating ultra-relativistic nucleus-nucleus collisions. In the non-perturbative realm, Monte Carlo simulations on the lattice relate the string tension to the temperature necessary to induce a first order phase transition from hadrons to QGP.34 These Monte-Carlo simulations give rise to the following estimates for $T_{c}$ the critical temperature $.205 \mathrm{GeV} \leq T_{\varepsilon} \leq .260 \mathrm{GeV}$. These estimates are made in the quenched approximation (the infinite quark mass limit). When massless quarks are assumed, which is more appropriate for the real world since quarks have very small masses, then $T_{c}$ is given by $.170 \mathrm{GeV} \leq T_{c} \leq .180 \mathrm{GeV}^{6}$ The critical temperature is lowered significantly.

Hydrodynamical models also exist which make a prediction for various quantities that are associated with the QGP. ${ }^{7}$ One such quantity that hydrodynamical models predict is $\boldsymbol{T}_{\mathbf{0}}$, the temperature of the subnuclear matter created in a relativistic nucleus-nucleus collision. Hence Brookhaven's R.H.I.C. will provide the opportunity to test hydrodynamical models of the formation of the QGP as well as the thermodynamic models of the subsequent particle production by the QGP.

In this letter we will use the hydrodynamical model developed by J. Cleymans, $\mathbf{R}$. V. Gavai and E. Suhonen ${ }^{7}$ to estimate $T_{0}$ in order to see whether it falls within the range for $T_{c}$ and then, based upon this predict prociuction rates for large mass muon pairs using the thermodynamical model that is called the fireball model. We then compare these rates to the perturbative quantum chromodynamical rates. In another paper we are attempting to extend the formalism of reference 7 to treat colliding proton beams.

In section II we state the essential results of the hydrodynamical model that we use to calculate $T_{0}$. 
In section III we compute the thermodynamical contribution to large mass muon pair production, whereas in section IV we compute the QCD rates.

Section $V$ gives comparisons between the thermal and perturbative $Q C D$ rates and conclusions.

This paper differs from other works on large mass dimuon production in several ways. One difference is that we make a definite prediction for $T_{0}$ and $\epsilon_{0}$ via the hydrodynamical model of reference 7. We also estimate the lifetimes of the fireballs, for each mass number and energy/nucleon, finding that they are non-trivial so that the temperature of the fireball evolves temporally according to an evolution equation that we develop. Hence the variation of the temperature with respect to proper time must be taken into account. Another difference is that we treat colliding beams. So, for colliding beams, we test the hydrodynamical and thermodynamical models as well as perturbative QCD.

\section{RESULTS FROM THE HYDRODYNAMICAL MODEL}

The formalism to compute $T_{0}$ and $\epsilon_{0}$, the temperature and energy density of the subnuclear matter created in an ultra-relativistic nucleus-nucleus (proton-proton) collision was derived in previous works. ${ }^{7.8}$ Here we will simply state the results of these considerations. The energy density of the subnuclear matter created in an ultra-relativistic nucleus-nucleus collision is given by

$$
\epsilon_{0}=B+\left[\frac{1.6}{A_{\perp} \tau_{0}} \frac{\widetilde{d N}}{d y}\right]^{1 / 3},
$$

where $B=0.4 \mathrm{GeV} / \mathrm{fm}^{3}$ and other physical quantities are explained and defined in reference 8. The temperature $T$ of the sub-nuclear matter created in an ultra-relativistic nucleus-nucleus collision is given by

$$
T=\left[\frac{\epsilon_{0}-B}{\lambda}\right]^{1 / 4}\left(J_{0} / r\right)^{1 / 3}
$$

where $\lambda$ is dependent on the composition of the sub-nuclear matter, i.e. $\lambda=12.17$ if one has a free gas of $u$ and $d$ quarks as well as gluons which is what one expects at temperatures around the critical temperature $T_{c-}$. Note that a useful boundary condition is that when $r=\tau_{0}$ then $T=T_{0}$. The various quantities in Eqs. (1) and (2) are tabulated in 
Table 1 for various species of ions that will be used in experiments in Brookhaven National Laboratory's R.H.I.C. project.

\section{THE THERMAL CONTRIBUTION}

The information which is tabulated in Table 1 warrants several comments. The last two columns contain several asterisked entries. The asterisked entries are valid for central collisions only. In this paper we define a central collision to be a small impact parameter collision $(|b| \approx 0)$. We may do this in the same sense that experimentalists can trigger on $|b| \approx 0$ collisions. In general a physical observable which,is related to particle collisions is averaged over all impact parameters. Howrever, the averaging process can hide essential physics as it does in the first two entries of the last two columns of Table 1. The energy density and temperature calculated for central collisions show that the phase transition from hadrons to QGP will take place if Montc-Carlo simulations are to be trusted in this context. On the other hand, the impact parameter averaged quantities hide this information. Thermal production will take place since the temperature necessary for hadronization has been achieved for central collisions for deuteium and carbon ions. However only one collision in six is a central collision so when comparing the rapidity densities the deuterium and carbon rates should be suitably adjusted. This adjustment is made by using the correct Boltzmann factor. The impact parameter averaged temperature should be used in that this contains the temperature for central collisions as well as the temperature for non-central collisions, each suitably weighted so that one gets the impact parameter averaged value. The reason that this works is as follows: In equation (1) $\frac{\widetilde{T}}{4}$ is an impact parameter averaged quantity so $\epsilon_{0}-B \propto\left[\frac{\pi}{\omega}\right]^{1 / 3}$. Now for central collisions we must insert a factor of $\frac{9}{2}$ whereas for non-central collisions the factor is $\frac{1}{4}, 2.9$ In order to get $\frac{\widetilde{2 N}}{d y} \equiv \frac{1}{\sigma_{t}} \frac{\tilde{d_{0}}}{d y}$, where $\sigma_{t} \equiv$ total inelastic cross-section, we make the following observation: Let $x$ be the probability that a collision is head-on (central) so that $1-x$ is the probability that the collision is not head-on (non-central). Then $\frac{\pi}{4} \approx \frac{1}{*}\left[x \frac{2}{2} \frac{\pi}{4}+(1-x) \frac{1}{4} \frac{\pi 0}{4}\right]$ so that

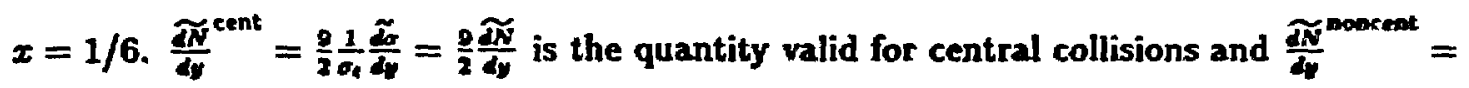
$\frac{1}{4} \frac{1}{\sigma_{t}} \frac{\tilde{\sigma}}{d y}=\frac{1}{4} \frac{\widetilde{N}}{d y}$ is valid for non-central collisions. Hence we see that in equation $(1) \epsilon_{N}-B$ is 
an impact parameter averaged quantity and therefore the temperature $T$ in equation (2) is also. When in doubt about the occurrence of a phase transition one should always compute $\frac{\widetilde{T}^{c e n t}}{d^{c}}$ and estimate $T_{0}$ for central collisions to see whether it falts in the prescribed range.

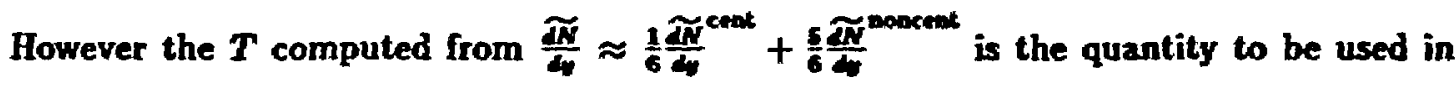
the Boltzmann factor. This gives the correct impact-parameter averaged rates. The other entries in Table 1 show that the ions in question will undergo a phase-transition evenwhen to $_{0}$ and $\boldsymbol{T}_{0}$ are impact-parameter averaged so the rapidity densities rising from them do not have to be adjusted. We proceed then to calculate the thermal rates.

The rate of emission of dimuons having mass $M$, energy $E^{*}$ and momentum $P^{\prime}$ in the rest frame of the fireball is given by

$$
\frac{E^{d} d N}{d M^{2} d^{3} P^{2} d^{4} x}=\frac{\alpha^{4}}{8 \pi^{4}} \sum_{a=\infty, d, e} e_{a}^{2} \exp \left[-E^{4} / T\right]
$$

$T$ is the temperature at which the dimuons are created. A transformation is made to the senter of mass frame by recalling the well known formula

$$
E^{\prime}=\sqrt{M^{2}+P_{T}^{2}} \cosh \left(y-y_{a}\right)
$$

where it will be recalled that $y$ is the muon pair rapidity and $y_{B}$ is the fireball rapidity in the center of mass frame. At this point we depart from the treatments of other authors in that we use an evolution equation for $T$ when integrating over the proper time component of $d^{4} x$. Recall that $d^{4} x=d t d^{3} x=r d r d^{3} x$. The reason for using the evolution equation for $t$ is that the fireballs that are produced have non-trivial lifetimes so that their temperatures evolve temporally. The lifetimes of the fireball are given in Table 2. In Table 2 the asterisked quantities follow our earlier convention. In addition it should be understood that lifetimes that have been averaged over all impact parameters are much less than those computed for central collisions. So all lifetimes are non-trivial in Table 2 . In the first two rows of the third column we have calculated the lifetimes of the fireballs created in head on (central) collisions. We can see that they are very large. The remaining entries are the impact parameter averaged quantities. However one should keep in mind that one collision in six is head on. Hence we have put "greater than" signs to indicate that $\tau_{f}$ is much larger than the exhibited entries. The basic assumption that we make here is that the times of physical interest are from to $(\sim 1 \mathrm{fm})$, the time for the nuclei to hadronize to 
$T_{f}$ the time where $T=0.140 \mathrm{GeV}$ and the fireball ceases to be highly excited and in fact either fragments or undergoes a phase transition, becoming a deformed nucleus. In this formulation when $\int d^{4} x$ is calculated the term $\sqrt{3} A^{\prime 1 / 3} \frac{V_{4}}{3.5} \frac{5 T_{n}}{M}$ originates in a very natural way. The evolution equation derives from Eq. (2):

$$
T=T_{0}\left(\frac{T_{0}}{T}\right)^{1 / 3}
$$

Next we perform the usual integrations over $\mathbb{S}^{\mathrm{s}} \mathrm{x}$ and over the transverse momentum, writing $T_{0}$ in terms of $A$, the mass number, then recasting $\rho\left(M^{2}, y\right)$ in a suitable form for comparison to $\rho_{\text {D.Y. }}\left(M^{2}, y\right)$. The result of these considerations is that

$$
\begin{aligned}
& 10^{8} M^{4} A^{1-4 / 3} \rho_{\text {thermal }}\left(M^{2}, y\right)=1.07 \times 10^{4} \sum_{n=0}^{4} \frac{T_{0}^{2}}{(5-n) !} \frac{T_{0}^{n-1}}{M^{n-3}}\left[\frac{\frac{M}{T_{R}} \cosh \left(y-y_{B}\right)-(n+1)}{\cosh ^{n+3}\left(y-y_{B}\right)}\right] \\
& \times \exp \left(-\frac{M}{T_{0}} \cosh \left(y-y_{B}\right)\right)[\mathrm{GeV}]^{-2},
\end{aligned}
$$

several terms having been dropped since they make a negligible contribution. In the fragmentation region it is well known that $\cosh \left(y-y_{B}\right) \approx \frac{1}{2}\left(\frac{x_{E}}{x_{-1}}+\frac{x_{m}}{x_{F}}\right)$ where $x_{F}=\frac{E}{E_{\max }}$ and $x_{M}=\frac{M}{E_{-4}} \frac{\gamma_{0}}{2 \gamma_{F}}$. For our beam energies $E_{\max } \approx 30 \mathrm{GeV}, \gamma_{0}$ and $\gamma_{F}$ were chosen to be 30 and 2 respectively. More complete definitions of the above quantities can be found in Ref. 8 (the original preprint). Figure 1 is a plot of $\log \left(10^{4} M^{4} A^{1-4 / 3} p_{\text {thermal }}\left(M^{2}, y\right)\right)$ vs. $x_{F}$ for various ions and masses of the muon pair. Figure 2 is a plot of $\log \left(10^{2} M^{4} A^{1-4 / 3}\right.$ pabermul $\left.\left(M^{2}, y\right)\right)$ vs $x_{F}$ for various ions, the muon pair having mass $M=4 \mathrm{GeV}$.

\section{THE PERTURBATIVE QUANTUM CHROMODYNAMICAL CONTRIBUTION}

We are interested in the inclusive process $A+A^{\prime} \rightarrow \mu^{+}+\mu^{-}+X$. The sub-process that's of relevance is $q+\bar{q} \rightarrow \mu^{+}+\mu^{-}$. Since the muons and quarks are elementary the quark counting rules tell us that we should expect

$$
\frac{d \sigma}{d t d y}\left(q+\bar{q} \rightarrow \mu^{+}+\mu^{-}\right) \sim \frac{1}{s^{2}} f(t / s)
$$


We must also take the mass number dependence into account. Present indications are that the strueture functions of partons within the nucleus differ from those within the nucleon in the following way: ${ }^{10}$

$$
x f^{N}\left(x, Q^{2}\right)=A x f^{n}\left(x, Q^{2}\right)+\Delta\left(x, Q^{2}\right),
$$

where $N$ refers to nuclear quantities, $n$ to nucleon quantities and $A$ is the mass number associated with the nucleus under consideration. The correction term $\Delta\left(x, Q^{2}\right)$ is associated with the motion of the core. Neglecting the motion of the core the inclusive cross-section is given by

$$
\frac{d \sigma}{d M^{2} d y}\left(A+A^{\prime} \rightarrow \mu^{+}+\mu^{-}+X\right)=\frac{4}{3} \pi \alpha^{2} \frac{A A^{\prime}}{M^{4}} \int d X_{1} \int d X_{2} \sum_{a} e_{a}^{2}\left[f_{a}^{A}\left(X_{1}\right) f_{2}^{A}\left(X_{2}\right)+\left(X_{1} \leftrightarrow X_{2}\right)\right],
$$

where $X_{1}=M e^{y} / \sqrt{s}$ and $X_{2}=M e^{-v} / \sqrt{s}, y$ being the rapidity of the pair.

The rapidity density becomes

$$
\begin{aligned}
\rho \mathrm{DY}\left(M^{2}, y\right) & \equiv \frac{d N}{d M^{2} d y}=\frac{1}{\sigma_{M^{\prime}}} \frac{d \sigma}{d M^{2} d y} \\
& =\frac{4}{3} \alpha^{2} \frac{1}{M^{4}} \frac{A A^{\prime}}{\left(1.1 A^{1 / 3}+1.1 A^{1 / 3}\right)^{2}} \int d X_{1} \int d X_{2} \sum_{a} e_{a}^{2}\left[f_{A}^{A}\left(X_{1}\right) f_{A}^{A^{\prime}}\left(X_{2}\right)+\left(X_{1} \leftrightarrow X_{2}\right)\right] .
\end{aligned}
$$

Here $R_{A^{\prime}}=\left(1.1 A^{1 / 3}+1.1 A^{1 / 3}\right)$ fm.

In units of mass in $\mathrm{GeV}$ we arrive at

$$
\begin{aligned}
& 10^{8} M^{4} A^{-1 / 3} \rho_{D Y}\left(M^{2}, y\right)=2.677 \int d X_{1} \int d X_{2} \sum_{a=x, d, A} e_{a}^{2}\left[f_{a}^{A}\left(X_{1}\right) f_{a}^{A}\left(X_{2}\right)+\left(X_{1} \leftrightarrow X_{2}\right)\right](G e V)^{-2} \\
& x_{F=1} \frac{9}{2}\left\{5.0\left(1-x_{F}\right)^{3.20578}-3.66\left(1-x_{F}\right)^{4.57376}\right. \\
& +.43\left(1-x_{F}\right)^{5.217}-.0019\left(1-x_{F}^{5.378}+.537\left(1-x_{F}\right)^{4.100}\right. \\
& \left.+.437\left(1-x_{F}\right)^{6.535}=.836\left(1-x_{F}\right)^{6.568}+.176\left(1-x_{F}\right)^{6.7334}+.0104\right\}(G e V)^{-2} \text {, }
\end{aligned}
$$

where the $9 / 2$ is the geometrical correction factor accounting for the $b=0$ trigger for a plasma consisting of up and down quarks as well as gluons. ${ }^{1,9}$ Figure 3 is a graph of $10^{2} M^{4} A^{p-1 / 3} \rho_{D Y}\left(M^{2}, y\right)$ versus $x_{F}$. Note that as $x_{F} \rightarrow 1, \rho_{D Y} \rightarrow 0$. 


\section{COMPARISONS, CONCLUSIONS}

In Figure 4, the Drell-Yan and thermal rates are plotted versus $x_{F}$. We have chosen to plot the thermal rates for a variety of ions. The mass of the exiting dimuons equals $4 \mathrm{GeV}$. The temperature of the sub-nuclear matter created in a collision between 2 such ions is fixed once the energy per nucleon and the mass number are known. Hence we make definite predictions for $p_{\text {thermal }}\left(M^{2}, y\right)$. From Fig. 4 it can be seen that although the DrellYan rate dominates convincingly in the small $x$ region, as $x_{p} \rightarrow 1^{-}$the thermal rates begin to dominate. At $x \boldsymbol{r} \geq 1$ the thermal rates peak and provide the only mechanism for dimuon production. The region $x_{F}>1$ is kinematically inaccessible to the Q.C.D. sub-processes. Hence we should look for dimuon pairs with $x \boldsymbol{z} \geq 1$ as a signal that the quark-gluon plasma has been produced. This signal should stand out very clearly-

In conclusion, in this paper, we have tested the predictions of a hydrodynamical as well as a fireball model for ultra-relativistic heavy ion collisions. If the basic assumptions that have been made in this paper are correct then we should be able to see manifestations of the quark-gluon plasma via dimuon pair production in the $x p \geq 1$ region of phase space. This should be a very clear signal. We expect smaller mass pairs to be favored energetically. Hence high energy small mass pairs ( $M \sim 2--4 \mathrm{GeV})$ will be the dominant signal.

\section{REFERENCES}

1. K. Kajantie and H. Miettinen, Z. Phys. C14, 357 (1982).

2. R. Anishetty, P. Koehler and L. McLerran, Phys. Rev. D22, 2793 (1980).

3. M. Creutz, Phys. Rev. Lett. 13, 553 (1979).

4. M. Creutz, Phys. Rev. D21, 2308 (1980).

5. G. Bhanot and C. Rebbi, Nucl. Phys. B180, [FS2] 469 (1981).

6. S. A. Gottlieb, W. Liu, D. Toussaint and R. L. Sugar, Phys. Rev. Lett. 59, 1513 (1987). 
7. J. Cleymans, R. V. Gavai and E. Suhonen, Physics Reports 130, 217 (1986).

8. C. Carney and L. E. Roberts, manuscript in preparation. This is a revised version of ANL-HEP-PR-87-85, August 7, 1987 by L. E. Roberts which contains the basic definitions.

9. L. E. Roberts, Nuovo Cimento A92, 2747 (1986).

10. G. Brown, "A-Dependence of the Drell-Yan Process", to be published in the proceedings of the Topical Conference on Nuclear Chromodynamics, held at Argonne National Laboratory, May 19-21, 1988.

\section{DISCLAIMER}

This repon was prepured as an acoowet of work spowored by an aneacy of the Unied Stues

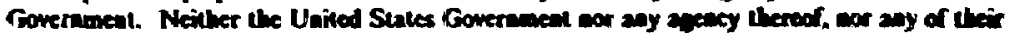

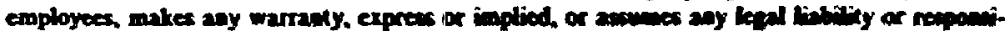

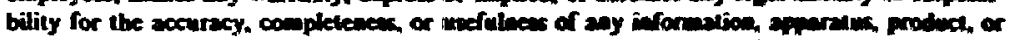

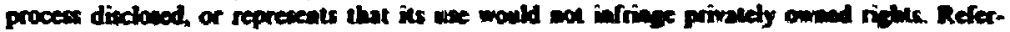

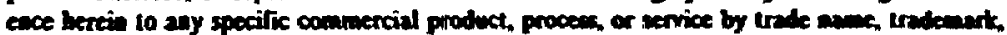

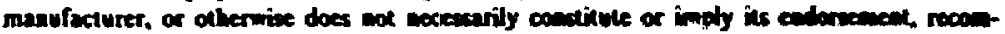

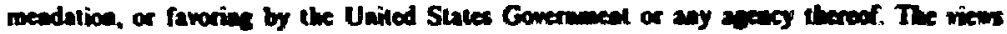

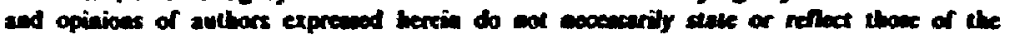

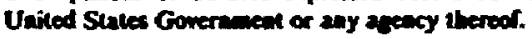


Table 1. Relevant data for the ion species that we've treated in this letter.

\begin{tabular}{|c|c|c|c|c|c|}
\hline Mass\# A & $\sqrt{s} /$ nucleon & $\tilde{d N} / d y$ & $A_{\perp}\left(\mathrm{fm}^{2}\right)$ & $\epsilon_{0}\left(\mathrm{GeV} / \mathrm{fm}^{3}\right)$ & $T_{0}(\mathrm{GeV})$ \\
\hline Deut-Deut & & & & $4.4^{\circ}$ & $0.2231^{\bullet}$ \\
\hline 2 & $125 \mathrm{GeV}$ & 8 & 6 & 0.93 & 0.135 \\
\hline Carbon-Carbon & & & & $9.16^{*}$ & $0.2714^{*}$ \\
\hline 12 & $125 \mathrm{GeV}$ & 48 & 20 & 1.6 & 0.165 \\
\hline \multicolumn{6}{|l|}{ Sulfur-Sulfur } \\
\hline 32 & $125 \mathrm{GeV}$ & 128 & 38 & 2.25 & 0.184 \\
\hline \multicolumn{6}{|l|}{$\mathbf{C U}-\mathbf{C U}$} \\
\hline 64 & $115 \mathrm{GeV}$ & 254 & 61 & 2.86 & 0.198 \\
\hline \multicolumn{6}{|l|}{ Iodine-Iodine } \\
\hline 127 & $104 \mathrm{GeV}$ & 508 & 96 & 3.8 & 0.214 \\
\hline \multicolumn{6}{|l|}{ Gold-Gold } \\
\hline 197 & $100 \mathrm{GeV}$ & 788 & 129 & 4.5 & 0.225 \\
\hline
\end{tabular}


Table 2. The lifetime $\tau_{\boldsymbol{g}}$ of the firebali created, assuming equal ion collisions, for the species treated in this letter and the energy per nucleon for each species. Note that as the mass number increases so does the lifetime of the fireball (assuming nearly fixed energy per nucleon).

\begin{tabular}{|c|c|c|}
\hline Mass\#A & $(\sqrt{5} /$ nucleon $) \mathrm{GeV}$ & $r_{f}(\mathrm{fm})$ \\
\hline Deut-Deut & & \\
\hline 2 & $125 \mathrm{GeV}$ & $4.04^{*}$ \\
\hline Carbon-Carbon & & \\
\hline 12 & $125 \mathrm{GeV}$ & $7.25^{\circ}$ \\
\hline Sulfur-Sulfur & & \\
\hline 32 & $125 \mathrm{GeV}$ & $>227$ \\
\hline CU-CU & & \\
\hline 64 & $115 \mathrm{GeV}$ & $>2.83$ \\
\hline Iodine-Iodine & & \\
\hline 127 & $104 \mathrm{GeV}$ & $>3.57$ \\
\hline Gold-Gold & & \\
\hline 197 & $100 \mathrm{GeV}$ & $>4.15$ \\
\hline
\end{tabular}




\section{FIGURE CAPTIONS}

Fig. 1. $\log \left(10^{8} M^{4} A^{1-4 / 3}\right.$ pthermal $_{\left.\left(M^{2}, y\right)\right)}$ is plotted vs. $x_{\text {r. }}$. Note the non-zero rates at $x_{F} \geq 1$. For thermal rates the key is a follows:

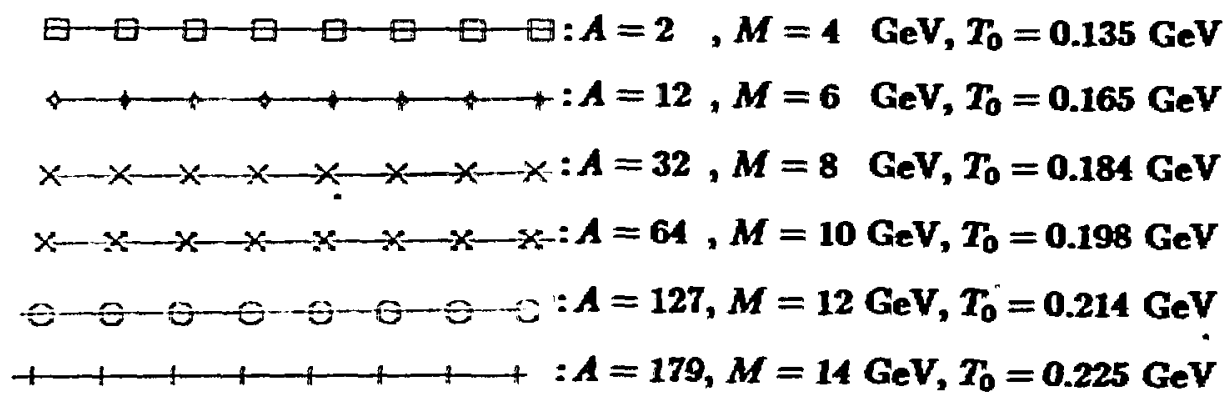

Fig. 2. Plot of $\log \left(10^{8} M^{4} A^{1-4 / 3} \rho_{t h}\left(M^{2}, y\right)\right)$ vs. $x_{F}$ for various ions. The mass of the muon pairs $M=1 \mathrm{GeV}$. The key is the same as in figure 1 for the mass numbers and temperatures.

Fig. 3. $\log \left(10^{8} M^{4} A^{1-1 / 3}\right.$ idy $\left.\left(M^{2}, y\right)\right)$ is plotted vs. $x_{F}$. Note that the kinematical region $x_{F} \geq 1$ is inaccessible to the Drell-Yan process.

Fig. 3. Drell-Yan rate vs. $x_{F}$. Note the region $x_{F} \geq 1$.

Fig. 4. Drell-Yan and thermal rates are compared vs. $x_{F}$. Once again note the region $x_{F} \geq 1$. 差 represents Drell-Yan rates. the thermal rates are represented by: $x-x-\infty \quad M=$ $4 \mathrm{GeV}, \longrightarrow M=3 \mathrm{GeV}$ and $\square M=2 \mathrm{GeV}$. 


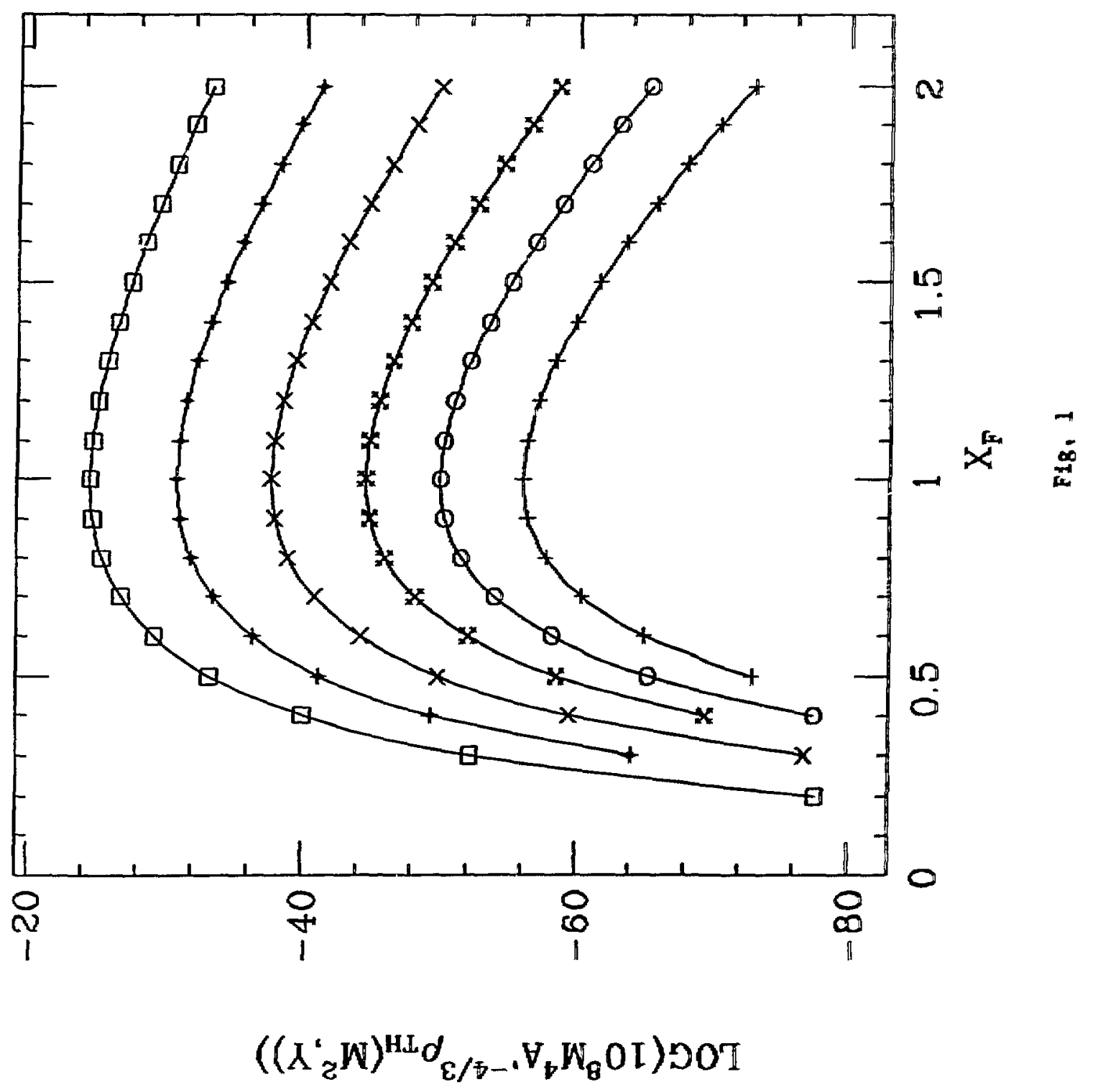




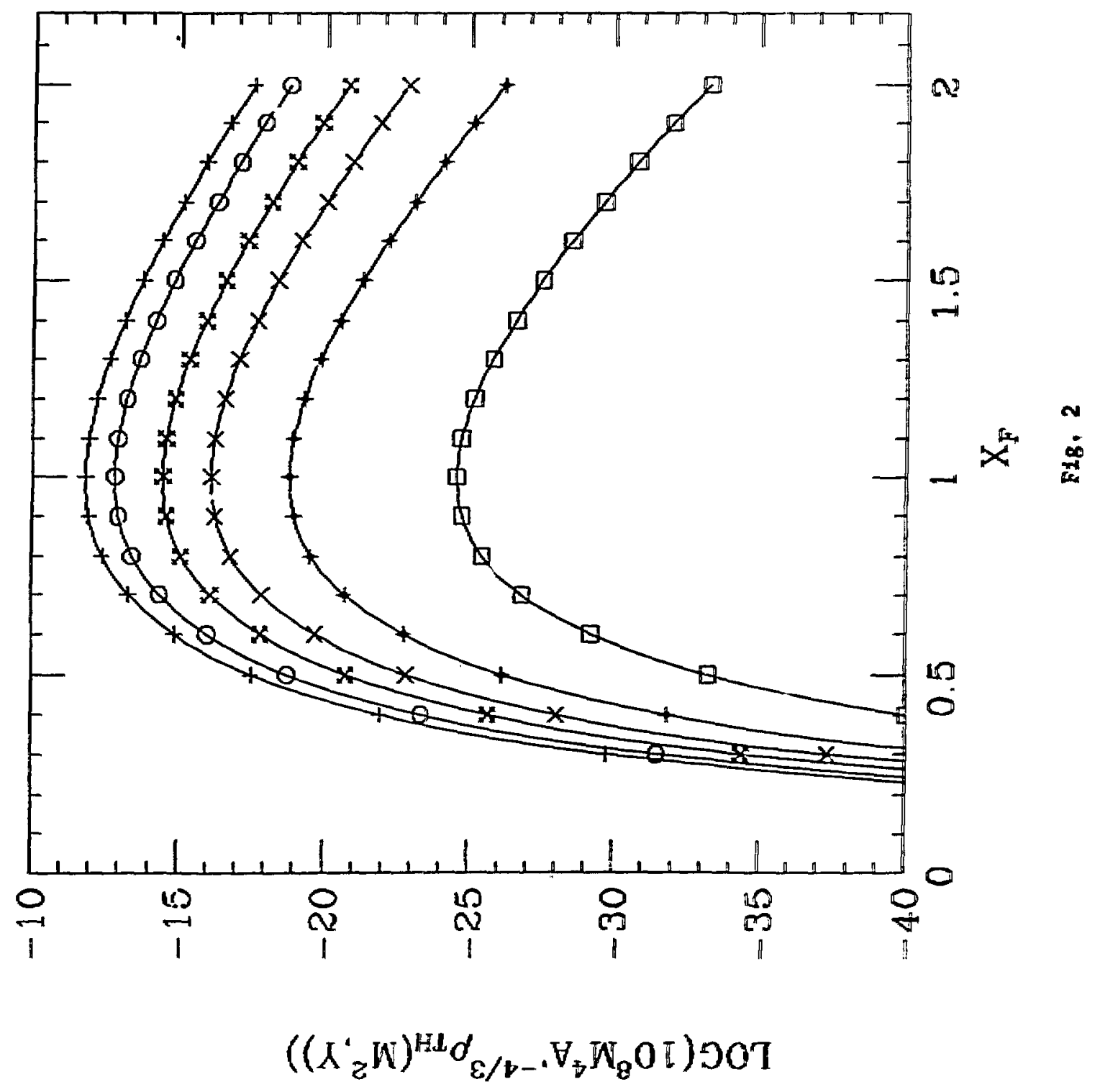




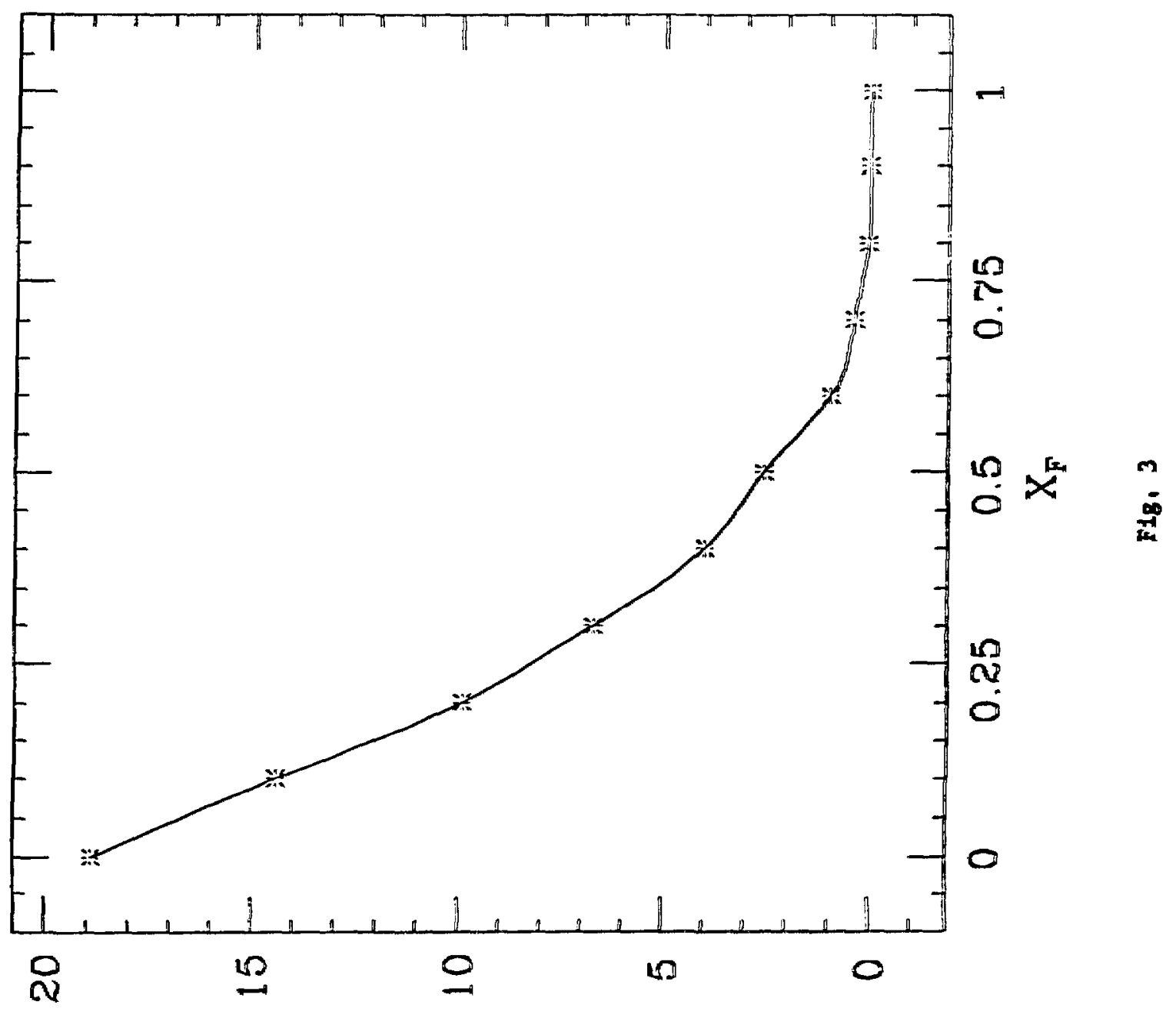

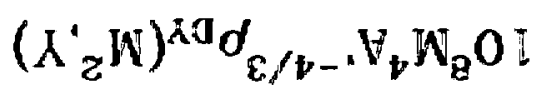




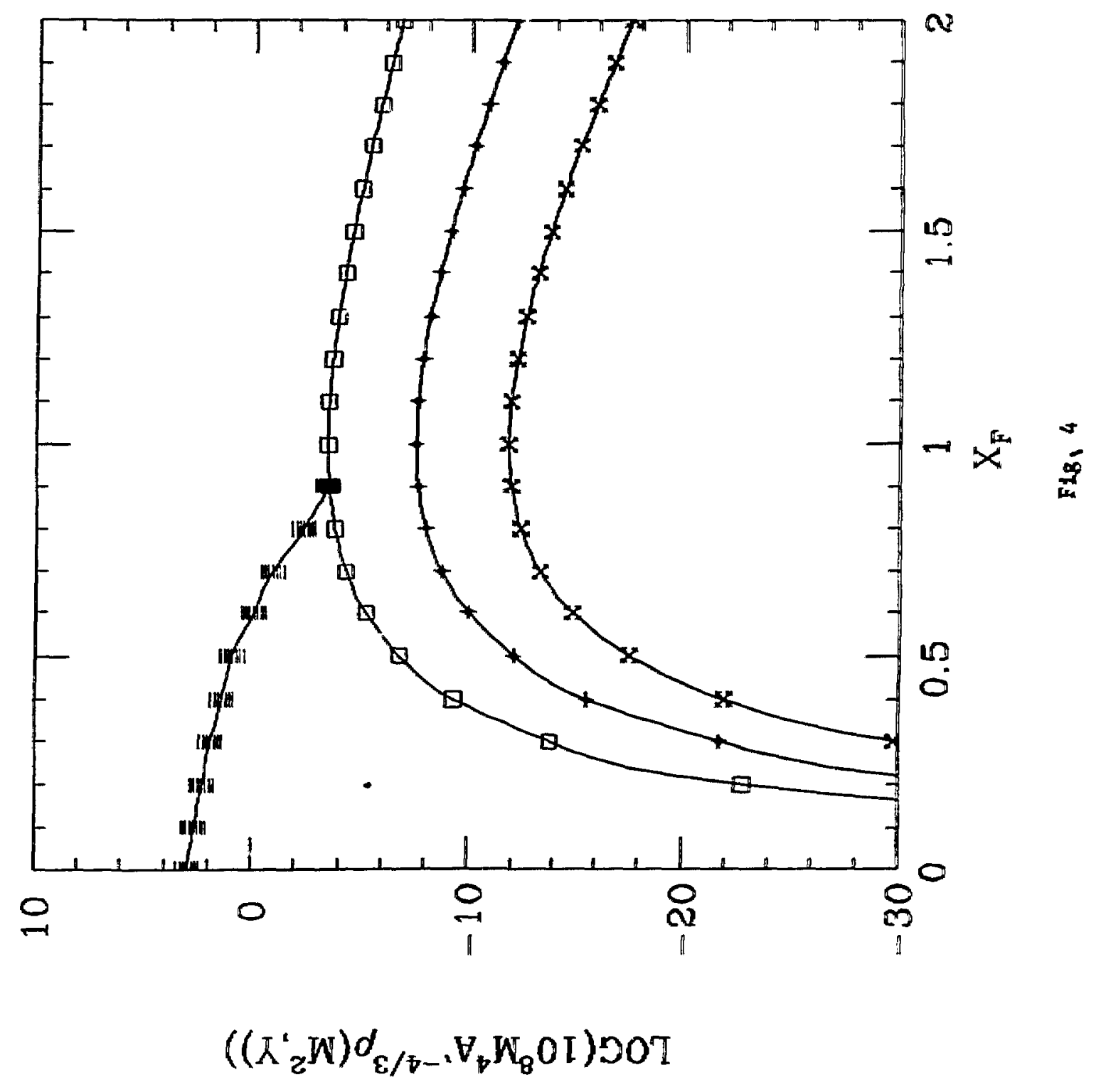

